\title{
Elastodynamic response of an anisotropic medium due to a line-load
}

\author{
N. R. Garg ${ }^{1}$, Anita Goel ${ }^{1}$, Aseem Miglani ${ }^{1}$, and Rajneesh Kumar ${ }^{1,2}$ \\ ${ }^{1}$ Department of Mathematics, Maharshi Dayanand University, Rohtak-124001, India \\ ${ }^{2}$ Department of Mathematics, Kurukshetra University, Kurukshetra-136 119, India \\ (Received November 6, 2002; Revised February 24, 2004; Accepted February 24, 2004)
}

\begin{abstract}
Two-dimensional elastodynamic displacements and stresses for a monoclinic solid have been obtained in relatively simple form by the use of the eigenvalue method, following Laplace and Fourier transforms. The main aim of this paper is to present a straightforward analytical eigenvalue method for a monoclinic solid which avoids the cumbersome nature of the problem and is convenient for numerical computation. The use of matrix notation avoids unwieldy mathematical expressions. A particular case of normal line-load acting in an orthotropic solid is discussed in detail. The corresponding deformation in time-domain is obtained numerically. The variations of elastodynamic displacements and stresses for an anisotropic medium with the horizontal distance have been shown graphically. It has been found that anisotropy is affecting the trend of distribution curves significantly.

Key words: Monoclinic, orthotropic, eignevalue method, two-dimensional deformation integral transforms, elastodynamic.
\end{abstract}

\section{Introduction}

The elastodynamic displacement response of an unbounded homogeneous linearly elastic solid to a line-load varying with time as a Dirac delta function provides a foundation to solve for the response to more general two-dimensional distributions of body forces and/or distributions of dislocations. The displacement Green's functions for a homogeneous isotropic linearly elastic solid are classical results in elastodynamic theory. The three-dimensional time-domain result has been obtained, amongst others, by Stokes (1849), Lamb (1904), Love (1944), Maruyama (1963), Achenbach (1973), Niwa et al. (1980), Karabolis and Beskos (1984). The dislocation problems of shear and tensile faults within the earth have received great attention in the geophysical literature from many investigators, e.g., Maruyama (1966), Okada (1985, 1992), Bonafede and Rivalta (1999a, b). The post-seismic deformation of a spherical, self-gravitating, stratified earth models has been extensively discussed by Piersanti et al. (1995), and others.

The corresponding solutions for anisotropic solids have also received a great deal of attention in the past. The generalization of the solution to include anisotropy is, however, very difficult. For a transversely isotropic medium, some relevant contributions are those of Buchwald (1959), Payton (1983), Yeatts (1984), Mura (1987) and Pan (1989).

The work by Lighthill (1960) for asmyptotic solution and by Duff (1960) on integral expressions for the Cauchy problem are the early treatments on general anistropy by the use of Fourier transform. Among the other relevant contributions for anisotropic solids, we mention those of Burridge (1967), Tverdokhlebov and Rose (1988), Wang and Achen-

Copy right(c) The Society of Geomagnetism and Earth, Planetary and Space Sciences (SGEPSS); The Seismological Society of Japan; The Volcanological Society of Japan; The Geodetic Society of Japan; The Japanese Society for Planetary Sciences; TERRAPUB. bach $(1992,1993,1994)$. Most of the solutions obtained in these papers are based on the use of a suitable combination of Radon transform and/or Fourier and cosine transforms.

Garg et al. (1996) obtained the representation of seismic sources causing static antiplane strain deformation of an orthotropic medium. The corresponding static plane strain deformation of an orthotropic elastic medium has been discussed by Garg et al. (2003). In the present paper a novel analytical eigenvalue method is presented for a monoclinic solid. The application of Laplace and Fourier transforms to the equations of motion for plane strain deformation of a monoclinic solid reduces them into a single linear homogeneous vector-matrix differential equation of second order, on which an eigenvalue method is applied to obtain a solution in the transformed domain. The procedure developed in this paper is relatively simple, straightforward, avoids the cumbersome nature of the problem and convenient for numerical computation. The form of the single governing equation for a monoclinic solid derived in this paper is new, which is obtained by a different approach. As a particular case, a normal line-load acting in the interior of an orthotropic elastic solid has been considered and the corresponding displacements and stresses in the time-domain have been obtained by inverting them numerically. The same method may also be applied to two-dimensional sources, such as strip-load. The variations of displacements and stresses for an anisotropic medium with the horizontal distance have been shown graphically. The deformation at any point of the medium is useful to analyse the deformation field around mining tremors and drilling into the crust of the earth. It may also find applications in various engineering problems, crystal physics and solid-earth geophysics regarding the deformation of an anisotropic solid. In fact, the study of a single force acting in a monoclinic solid forms a basis for further investigations. 


\section{Basic Equations and Theory}

The equations of motion in the Cartesian co-ordinate system $\left(x_{1}, x_{2}, x_{3}\right)$ for zero body force are

$$
\begin{aligned}
& \frac{\partial \tau_{11}}{\partial x_{1}}+\frac{\partial \tau_{12}}{\partial x_{2}}+\frac{\partial \tau_{13}}{\partial x_{3}}=\rho \frac{\partial^{2} u_{1}}{\partial t^{2}}, \\
& \frac{\partial \tau_{21}}{\partial x_{1}}+\frac{\partial \tau_{22}}{\partial x_{2}}+\frac{\partial \tau_{23}}{\partial x_{3}}=\rho \frac{\partial^{2} u_{2}}{\partial t^{2}}, \\
& \frac{\partial \tau_{31}}{\partial x_{1}}+\frac{\partial \tau_{32}}{\partial x_{2}}+\frac{\partial \tau_{33}}{\partial x_{3}}=\rho \frac{\partial^{2} u_{3}}{\partial t^{2}},
\end{aligned}
$$

where $\tau_{i j}(i, j=1,2,3)$ are the components of the stress tensor, $\left(u_{1}, u_{2}, u_{3}\right)$ are the components of the displacement vector and $\rho$ is the density of the elastic medium.

The stress-strain relations for a monoclinic elastic medium, with $x_{1} x_{2}$-plane as plane of elastic symmetry, are (Atanackovic and Guran, 2000)

$$
\begin{aligned}
& \tau_{11}=d_{11} e_{11}+d_{12} e_{22}+d_{13} e_{33}+2 d_{16} e_{12}, \\
& \tau_{22}=d_{12} e_{11}+d_{22} e_{22}+d_{23} e_{33}+2 d_{26} e_{12}, \\
& \tau_{33}=d_{13} e_{11}+d_{23} e_{22}+d_{33} e_{33}+2 d_{36} e_{12}, \\
& \tau_{23}=2 d_{44} e_{23}+2 d_{45} e_{13}, \\
& \tau_{13}=2 d_{45} e_{23}+2 d_{55} e_{13}, \\
& \tau_{12}=d_{16} e_{11}+d_{26} e_{22}+d_{36} e_{33}+2 d_{66} e_{12},
\end{aligned}
$$

where $e_{i j}(i, j=1,2,3)$ are the components of strain tensor and are related with displacement components $\left(u_{1}, u_{2}, u_{3}\right)$ through the relations

$$
e_{i j}=\frac{1}{2}\left[\frac{\partial u_{i}}{\partial x_{j}}+\frac{\partial u_{j}}{\partial x_{i}}\right], \quad 1 \leq i, j \leq 3 .
$$

The two-suffix symmetric quantities $d_{i j}(i, j=1,2, \cdots, 6)$ are the elastic moduli for the monoclinic elastic medium.

If the medium has three coordinate planes as the planes of elastic symmetry, the quantities $d_{i j}$ are the elastic moduli for the orthotropic elastic medium in which (Mase and Mase, 1999)

$$
d_{16}=d_{26}=d_{36}=d_{45}=0 .
$$

Further a transversely isotropic with $x_{3}$-axis coinciding with the axis of symmetry is a particular case of orthotropy in which

$$
d_{22}=d_{11}, \quad d_{23}=d_{13}, \quad d_{55}=d_{44}, \quad d_{66}=\frac{1}{2}\left(d_{11}-d_{12}\right) .
$$

Isotropy is also a particular case of an orthotropy in which

$$
\begin{gathered}
d_{11}=d_{22}=d_{33}=\lambda+2 \mu, \quad d_{12}=d_{13}=d_{23}=\lambda, \\
d_{44}=d_{55}=d_{66}=\mu,
\end{gathered}
$$

where $\lambda$ and $\mu$ are Lamé elastic moduli.

For convenience, we shall write $\left(x_{1}, x_{2}, x_{3}\right)=(x, y, z)$ and $\left(u_{1}, u_{2}, u_{3}\right)=(u, v, w)$.

The equations of motion for a monoclinic elastic medium in terms of displacement components can be obtained from
Eqs. (1)-(5). We find

$$
\begin{aligned}
& {\left[d_{11} \frac{\partial^{2} u}{\partial x^{2}}+d_{66} \frac{\partial^{2} u}{\partial y^{2}}+d_{55} \frac{\partial^{2} u}{\partial z^{2}}+2 d_{16} \frac{\partial^{2} u}{\partial x \partial y}\right]} \\
& +\left[d_{16} \frac{\partial^{2} v}{\partial x^{2}}+d_{26} \frac{\partial^{2} v}{\partial y^{2}}+d_{45} \frac{\partial^{2} v}{\partial z^{2}}+\left(d_{12}+d_{66}\right) \frac{\partial^{2} v}{\partial x \partial y}\right] \\
& +\left[\left(d_{13}+d_{55}\right) \frac{\partial^{2} w}{\partial x \partial z}+\left(d_{36}+d_{45}\right) \frac{\partial^{2} w}{\partial y \partial z}\right]=\rho \frac{\partial^{2} u}{\partial t^{2}}
\end{aligned}
$$

$$
\begin{aligned}
& {\left[d_{16} \frac{\partial^{2} u}{\partial x^{2}}+d_{26} \frac{\partial^{2} u}{\partial y^{2}}+d_{45} \frac{\partial^{2} u}{\partial z^{2}}+\left(d_{66}+d_{12}\right) \frac{\partial^{2} u}{\partial x \partial y}\right]} \\
& +\left[d_{66} \frac{\partial^{2} v}{\partial x^{2}}+d_{22} \frac{\partial^{2} v}{\partial y^{2}}+d_{44} \frac{\partial^{2} v}{\partial z^{2}}+2 d_{26} \frac{\partial^{2} v}{\partial x \partial y}\right] \\
& +\left[\left(d_{36}+d_{45}\right) \frac{\partial^{2} w}{\partial x \partial z}+\left(d_{23}+d_{44}\right) \frac{\partial^{2} w}{\partial y \partial z}\right]=\rho \frac{\partial^{2} v}{\partial t^{2}},
\end{aligned}
$$

$$
\begin{aligned}
& {\left[\left(d_{55}+d_{13}\right) \frac{\partial^{2} u}{\partial x \partial z}+\left(d_{45}+d_{36}\right) \frac{\partial^{2} u}{\partial y \partial z}\right]} \\
& +\left[\left(d_{45}+d_{36}\right) \frac{\partial^{2} v}{\partial x \partial z}+\left(d_{44}+d_{23}\right) \frac{\partial^{2} v}{\partial y \partial z}\right] \\
& +\left[d_{55} \frac{\partial^{2} w}{\partial x^{2}}+d_{44} \frac{\partial^{2} w}{\partial y^{2}}+d_{33} \frac{\partial^{2} w}{\partial z^{2}}+2 d_{45} \frac{\partial^{2} w}{\partial x \partial y}\right]=\rho \frac{\partial^{2} w}{\partial t^{2}} .
\end{aligned}
$$

We now consider the plane-strain deformation, parallel to the $x y$-plane, in which the displacement components are independent of $z$ and are of the type

$$
u=u(x, y, t), \quad v=v(x, y, t), \quad w=0 .
$$

We assume that the displacement components and their timederivatives are initially zero.

The non-zero stresses for the plane-strain problem (parallel to $x y$-plane) for a monoclinic medium are to be obtained from Eqs. (4) and (5). We find

$$
\begin{aligned}
\tau_{11} & =d_{11} \frac{\partial u}{\partial x}+d_{12} \frac{\partial v}{\partial y}+d_{16}\left(\frac{\partial u}{\partial y}+\frac{\partial v}{\partial x}\right), \\
\tau_{22} & =d_{12} \frac{\partial u}{\partial x}+d_{22} \frac{\partial v}{\partial y}+d_{26}\left(\frac{\partial u}{\partial y}+\frac{\partial v}{\partial x}\right), \\
\tau_{33} & =d_{13} \frac{\partial u}{\partial x}+d_{23} \frac{\partial v}{\partial y}+d_{36}\left(\frac{\partial u}{\partial y}+\frac{\partial v}{\partial x}\right), \\
\tau_{12} & =d_{16} \frac{\partial u}{\partial x}+d_{26} \frac{\partial v}{\partial y}+d_{66}\left(\frac{\partial u}{\partial y}+\frac{\partial v}{\partial x}\right) .
\end{aligned}
$$

The equations of motion, given in Eqs. (7)-(8), for planestrain deformation of a monoclinic elastic medium are

$$
\begin{aligned}
& {\left[d_{11} \frac{\partial^{2} u}{\partial x^{2}}+d_{66} \frac{\partial^{2} u}{\partial y^{2}}+2 d_{16} \frac{\partial^{2} u}{\partial x \partial y}\right]} \\
& +\left[d_{16} \frac{\partial^{2} v}{\partial x^{2}}+d_{26} \frac{\partial^{2} v}{\partial y^{2}}+\left(d_{12}+d_{66}\right) \frac{\partial^{2} v}{\partial x \partial y}\right]=\frac{\rho \partial^{2} u}{\partial t^{2}},
\end{aligned}
$$

$$
\begin{aligned}
& {\left[d_{16} \frac{\partial^{2} u}{\partial x^{2}}+d_{26} \frac{\partial^{2} u}{\partial y^{2}}+\left(d_{66}+d_{12}\right) \frac{\partial^{2} u}{\partial x \partial y}\right]} \\
& +\left[d_{66} \frac{\partial^{2} v}{\partial x^{2}}+d_{22} \frac{\partial^{2} v}{\partial y^{2}}+2 d_{26} \frac{\partial^{2} v}{\partial x \partial y}\right]=\frac{\rho \partial^{2} v}{\partial t^{2}}
\end{aligned}
$$


and equation (9) reduces to an identity.

We now reduce Eqs. (14) and (15) in the non-dimensional variable form. We define the following non-dimensional quantities

$$
u^{\prime}=\frac{u}{h}, \quad v^{\prime}=\frac{v}{h}, \quad x^{\prime}=\frac{x}{h}, \quad y^{\prime}=\frac{y}{h}, \quad t^{\prime}=\omega t,
$$

where $\omega=\sqrt{\frac{d_{66}}{\rho h^{2}}}$ is the "known frequency" and ' $h$ ' is a known fixed quantity which has the dimension of length. Let

$$
a=\frac{d_{11}}{d_{66}}, b=\frac{d_{22}}{d_{66}}, c=\frac{d_{12}}{d_{66}}, d=\frac{d_{26}}{d_{66}}, e=\frac{d_{16}}{d_{66}}
$$

be the dimensionless elastic moduli.

The field equations (14) and (15) in the non-dimensional form, after suppressing the dashes, are as

$$
\begin{aligned}
& {\left[a \frac{\partial^{2} u}{\partial x^{2}}+\frac{\partial^{2} u}{\partial y^{2}}+2 e \frac{\partial^{2} u}{\partial x \partial y}\right]} \\
& +\left[e \frac{\partial^{2} v}{\partial x^{2}}+d \frac{\partial^{2} v}{\partial y^{2}}+(c+1) \frac{\partial^{2} v}{\partial x \partial y}\right]=\frac{\partial^{2} u}{\partial t^{2}} \\
& {\left[e \frac{\partial^{2} u}{\partial x^{2}}+d \frac{\partial^{2} u}{\partial y^{2}}+(1+c) \frac{\partial^{2} u}{\partial x \partial y}\right]} \\
& +\left[\frac{\partial^{2} v}{\partial x^{2}}+b \frac{\partial^{2} v}{\partial y^{2}}+2 d \frac{\partial^{2} v}{\partial x \partial y}\right]=\frac{\partial^{2} v}{\partial t^{2}} .
\end{aligned}
$$

In Eqs. (18) and (19), $x$ is the dimensionless vertical distance, $y$ is the dimensionless horizontal distance. $u$ is the dimensionless vertical displacement component, $v$ is the dimensionless horizontal displacement component and $a, b, c$, $d, e$ are dimensionless elastic coefficients.

We define the Laplace transform $\hat{f}(x, y, p)$ of $f(x, y, t)$ by the relation (Debnath, 1995)

$$
L[f(x, y, t)]=\hat{f}(x, y, p)=\int_{0}^{\infty} e^{-p t} f(x, y, t) d t,
$$

where $p$ is the Laplace transform parameter. We know the following properties of Laplace transform.

$$
\begin{aligned}
L\left[\frac{\partial^{2}}{\partial t^{2}} f(x, y, t)\right]= & p^{2} \hat{f}(x, y, p)-p f(x, y, t=0) \\
& -\left[\frac{\partial}{\partial t} f(x, y, t)\right]_{t=0}, \\
L\left[\frac{\partial}{\partial x} f(x, y, t)\right]= & \frac{\partial \hat{f}}{\partial x}(x, y, p), \\
L\left[\frac{\partial}{\partial y} f(x, y, t)\right]= & \frac{\partial \hat{f}}{\partial y}(x, y, p) .
\end{aligned}
$$

The Laplace transform of Eqs. (18) and (19) gives

$$
\begin{aligned}
& {\left[a \frac{\partial^{2} \hat{u}}{\partial x^{2}}+\frac{\partial^{2} \hat{u}}{\partial y^{2}}+2 e \frac{\partial^{2} \hat{u}}{\partial x \partial y}\right]} \\
& +\left[e \frac{\partial^{2} \hat{v}}{\partial x^{2}}+d \frac{\partial^{2} \hat{v}}{\partial y^{2}}+(c+1) \frac{\partial^{2} \hat{v}}{\partial x \partial y}\right]=p^{2} \hat{u}, \\
& {\left[e \frac{\partial^{2} \hat{u}}{\partial x^{2}}+d \frac{\partial^{2} \hat{u}}{\partial y^{2}}+(1+c) \frac{\partial^{2} \hat{u}}{\partial x \partial y}\right]} \\
& +\left[\frac{\partial^{2} \hat{v}}{\partial x^{2}}+b \frac{\partial^{2} \hat{v}}{\partial y^{2}}+2 d \frac{\partial^{2} \hat{v}}{\partial x \partial y}\right]=p^{2} \hat{v} .
\end{aligned}
$$

The Fourier transform $\bar{f}(x, k, p)$ of $\hat{f}(x, y, p)$ is given by the relation

$$
\bar{f}(x, k, p) \equiv F[\hat{f}(x, y, p)]=\int_{-\infty}^{\infty} \hat{f}(x, y, p) e^{i k y} d y,
$$

so that

$$
\hat{f}(x, y, p)=\frac{1}{2 \pi} \int_{-\infty}^{\infty} \bar{f}(x, k, p) e^{-i k y} d k,
$$

where $k$ is the transformed Fourier parameter. We know the following properties of Fourier transform (Sneddon, 1951),

$$
\begin{gathered}
F\left[\frac{\partial^{2}}{\partial x \partial y} \hat{f}(x, y, p)\right]=(-i k) \frac{d}{d x} \bar{f}(x, k, p), \\
F\left[\frac{\partial^{2}}{\partial x^{2}} \hat{f}(x, y, p)\right]=\frac{d^{2}}{d x^{2}} \bar{f}(x, k, p), \\
F\left[\frac{\partial^{2}}{\partial y^{2}} \hat{f}(x, y, p)\right]=-k^{2} \bar{f}(x, k, p) .
\end{gathered}
$$

Applying the Fourier transformation on Eqs. (22) and (23), we obtain

$$
\begin{aligned}
& {\left[a \frac{d^{2} \bar{u}}{d x^{2}}-2 i e k \frac{d \bar{u}}{d x}-k^{2} \bar{u}\right]} \\
& +\left[e \frac{d^{2} \bar{v}}{d x^{2}}-i k(1+c) \frac{d \bar{v}}{d x}-k^{2} d v\right]=p^{2} \bar{u} \\
& {\left[e \frac{d^{2} \bar{u}}{d x^{2}}-i k(1+c) \frac{d \bar{u}}{d x}-k^{2} d \bar{u}\right]} \\
& +\left[\frac{d^{2} \bar{v}}{d x^{2}}-2 i d k \frac{d \bar{v}}{d x}-k^{2} b \bar{v}\right]=p^{2} \bar{v}
\end{aligned}
$$

The above two differential equations can be written in the following homogeneous vector-matrix differential equation

$$
A \frac{d^{2} N}{d x^{2}}+B \frac{d N}{d x}+C N=0
$$

where

$$
\begin{gathered}
A=\left[\begin{array}{ll}
a & e \\
e & 1
\end{array}\right], \quad B=\left[\begin{array}{cc}
-2 i e k & -i k(1+c) \\
-i k(1+c) & -2 i d k
\end{array}\right], \\
C=\left[\begin{array}{cc}
-p^{2}-k^{2} & -k^{2} d \\
-k^{2} d & -p^{2}-b k^{2}
\end{array}\right], \quad N=\left[\begin{array}{c}
\bar{u} \\
\bar{v}
\end{array}\right] .
\end{gathered}
$$

We note that matrices $A, B, C$ are all symmetric. Matrix $A$ depends upon dimensionless elastic moduli only while matrices $B$ and $C$ depend upon dimensionless wave number $k$. The governing equation (29) for a monoclinic solid is a new contribution.

We now assume a solution of matrix equation (29) of the form

$$
N(x, k)=E(k) e^{s x},
$$

where $s$ is a parameter and $E(k)$ is a matrix of the type $2 \times 1$.

Substitution of the value of $N$ from Eq. (31) into differential equation (29) gives the following characteristic equation

$$
a_{0} s^{4}+4 a_{1} s^{3}+6 a_{2} s^{2}+4 a_{3} s+a_{4}=0,
$$


where

$$
\begin{aligned}
& a_{0}=a-e^{2}, \\
& a_{1}=\frac{-i k(a d-c e)}{2}, \\
& a_{2}=\left\{-p^{2}(a+1)+k^{2}\left[-a b+c^{2}+2 c-2 e d\right]\right\} / 6, \\
& a_{3}=\left\{2 i k p^{2}(e+d)-2 i k^{3} c d+2 i e k^{3} b\right\} / 4, \\
& a_{4}=\left[p^{4}+p^{2} k^{2}(b+1)+k^{4}\left(b-d^{2}\right)\right] .
\end{aligned}
$$

To solve biquadratic equation (32), we make use of Descartes' method, in which we remove the second term by using the transformation

$$
z^{*}=a_{0} s+a_{1} .
$$

Then the equation (32) is transformed into

$$
\left(z^{*}\right)^{4}+6 H\left(z^{*}\right)^{2}+4 G z^{*}+\left(a_{0}^{2} I-3 H^{2}\right)=0,
$$

where

$$
\begin{aligned}
& H=a_{0} a_{2}-a_{1}^{2} ; G=a_{0}^{2} a_{3}-3 a_{0} a_{1} a_{2}+2 a_{1}^{3}, \\
& I=a_{0} a_{4}-4 a_{1} a_{3}+3 a_{2}^{2} .
\end{aligned}
$$

Following the Descartes' method, the left hand side of Eq. (35) can be resolved into a product of two quadratic factors as

$$
\begin{aligned}
z^{* 4} & +6 H z^{* 2}+4 G z^{*}+\left(a_{0}^{2} I-3 H^{2}\right) \\
& =\left(z^{* 2}+n z^{*}+q\right)\left(z^{* 2}-n z^{*}+q^{\prime}\right),
\end{aligned}
$$

where

$$
\begin{gathered}
q+q^{\prime}=n^{2}+6 H, \quad q q^{\prime}=a_{0}^{2} I-3 H^{2}, \\
n\left(q^{\prime}-q\right)=4 G .
\end{gathered}
$$

When $n=0$, then $q$ and $q^{\prime}$ are determined from first two equations in (38). Otherwise, eliminating $q$ and $q^{\prime}$ from three equations in (38), we get

$$
\alpha^{3}+3 b_{1} \alpha^{2}+3 b_{2} \alpha+b_{3}=0,
$$

where

$$
\begin{aligned}
& \alpha=n^{2}, \quad b_{1}=4 H, \\
& \quad b_{2}=\frac{4\left(12 H^{2}-a_{0}^{2} I\right)}{3}, \quad b_{3}=-16 G^{2} .
\end{aligned}
$$

Using the Cardan's method, the cubic equation (39) is reduced to

$$
\delta^{3}+3 H_{1} \delta+G_{1}=0,
$$

where

$$
\delta=\alpha+b_{1}, \quad H_{1}=b_{2}-b_{1}^{2}, \quad G_{1}=b_{3}-3 b_{1} b_{2}+2 b_{1}^{3} .
$$

The roots of the Eq. (41) are

$$
\delta=u_{0}+v_{0}
$$

where $u_{0}$ and $v_{0}$ are given by

$$
u_{0}^{3}=\frac{-G_{1}+\sqrt{G_{1}^{2}+4 H_{1}^{3}}}{2}, \quad v_{0}^{3}=\frac{-G_{1}-\sqrt{G_{1}^{2}+4 H_{1}^{3}}}{2},
$$

satisfying the condition

$$
u_{0} v_{0}=-H_{1} .
$$

Thus, $n$ is given by

$$
n^{2}=\delta-b_{1}
$$

and $q$ and $q^{\prime}$ are then to be calculated from Eqs. (38) and (46). From Eq. (37), we obtain

$$
\left.\begin{array}{l}
z_{1}^{*}=\frac{-n+\sqrt{n^{2}-4 q}}{2}, z_{2}^{*}=\frac{-n-\sqrt{n^{2}-4 q}}{2}, \\
z_{3}^{*}=\frac{n+\sqrt{n^{2}-4 q^{\prime}}}{2}, \quad z_{4}^{*}=\frac{n-\sqrt{n^{2}-4 q^{\prime}}}{2},
\end{array}\right\}
$$

as the roots of biquadratic equation (35). Hence the eigenvalues of the problem are $s_{1}, s_{2}, s_{3}, s_{4}$, where

$$
s_{L}=\frac{z_{L}^{*}-a_{1}}{a_{0}} \quad \text { for } L=1,2,3,4,
$$

which are usually distinct. The corresponding eigenvectors are

$$
X_{L}^{T}=\left[P_{L}, 1\right]
$$

where

$$
\begin{array}{r}
P_{L}=\frac{-\left[e s_{L}^{2}-i k s_{L}(1+c)-k^{2} d\right]}{a s_{L}^{2}-2 i k e s_{L}-p^{2}-k^{2}} \\
=\frac{-\left[s_{L}^{2}-2 i k d s_{L}-p^{2}-b k^{2}\right]}{e s_{L}^{2}-i k s_{L}(1+c)-k^{2} d} \\
\text { for } L=1,2,3,4,
\end{array}
$$

and $(\cdots)^{T}$ denotes the transpose of the matrix $(\cdots)$. Thus, a general solution of the vector-matrix differential equation (29) for a monoclinic elastic medium becomes

$$
N(x, k)=\sum_{L=1}^{4}\left(B_{L} X_{L} e^{s_{L} x}\right),
$$

where $B_{1}, B_{2}, B_{3}, B_{4}$ are constants to be determined from prescribed boundary conditions. The case of repeated eigenvalues can be tackled by making suitable modification in the expression on the right side of Eq. (51), as discussed by Ross (1984).

Equation (51) gives

$$
\bar{u}(x, k)=B_{1} P_{1} e^{s_{1} x}+B_{2} P_{2} e^{s_{2} x}+B_{3} P_{3} e^{s_{3} x}+B_{4} P_{4} e^{s_{4} x},
$$

$$
\bar{v}(x, k)=B_{1} e^{s_{1} x}+B_{2} e^{s_{2} x}+B_{3} e^{s_{3} x}+B_{4} e^{s_{4} x} .
$$

We define the dimensionless stresses by the following relations

$$
\tau_{11}^{\prime}=\frac{\tau_{11}}{d_{66}}, \quad \tau_{12}^{\prime}=\frac{\tau_{12}}{d_{66}}
$$

The expressions for the stress components in the nondimensional form (after suppressing the dashes) for the 
present two-dimensional plane-strain problem are calculated from Eqs. (10), (13), (17) and (54). We find

$$
\begin{aligned}
\tau_{11} & =\left(a \frac{\partial u}{\partial x}+c \frac{\partial v}{\partial y}+e \frac{\partial u}{\partial y}+e \frac{\partial v}{\partial x}\right), \\
\tau_{12} & =\left(e \frac{\partial u}{\partial x}+d \frac{\partial v}{\partial y}+\frac{\partial u}{\partial y}+\frac{\partial v}{\partial x}\right) .
\end{aligned}
$$

The Laplace-Fourier transformation of Eq. (55) gives

$$
\begin{aligned}
& \bar{\tau}_{11}=\left(a \frac{\partial \bar{u}}{\partial x}-i k c \bar{v}-i k e \bar{u}+e \frac{\partial \bar{v}}{\partial x}\right), \\
& \bar{\tau}_{12}=\left(e \frac{\partial \bar{u}}{\partial x}-i k d \bar{v}-i k \bar{u}+\frac{\partial \bar{v}}{\partial x}\right) .
\end{aligned}
$$

Putting the value of transformed displacements $\bar{u}$ and $\bar{v}$ from Eqs. (52) and (53) and their derivatives in Eq. (56), we find the following expressions for transformed stresses

$$
\begin{aligned}
& \bar{\tau}_{11}=\left[Q_{1} B_{1} e^{s_{1} x}+Q_{2} B_{2} e^{s_{2} x}+Q_{3} B_{3} e^{s_{3} x}+Q_{4} B_{4} e^{s_{4} x}\right], \\
& \bar{\tau}_{12}=\left[R_{1} B_{1} e^{s_{1} x}+R_{2} B_{2} e^{s_{2} x}+R_{3} B_{3} e^{s_{3} x}+R_{4} B_{4} e^{s_{4} x}\right],
\end{aligned}
$$

where

$$
\left.\begin{array}{c}
Q_{L}=a P_{L} s_{L}-i k c-i k e P_{L}+s_{L} e, \\
R_{L}=e P_{L} s_{L}-i k d-i k P_{L}+s_{L} .
\end{array}\right\} \text { for } L=1,2,3,4
$$

From now onwards, for the sake of simplicity, we are considering elastic materials which have orthotropic symmetry. For an orthotropic material, we have

$$
\begin{aligned}
& d=0, \quad e=0, \\
& a_{0}=a, \quad a_{1}=0 \\
& \left.a_{2}=\frac{\left[-p^{2}(a+1)+k^{2}\left(-a b+c^{2}+2 c\right)\right]}{6}\right\} \\
& a_{3}=0, \quad a_{4}=\left[p^{4}+p^{2} k^{2}(1+b)+b k^{4}\right], \\
& H=\frac{a}{6}\left[-p^{2}(a+1)-k^{2}\left(a b-c^{2}-2 c\right)\right] \\
& I=a\left[p^{4}+p^{2} k^{2}(1+b)+b k^{4}\right] \\
& \left.+\frac{1}{12}\left[-p^{2}(a+1)+k^{2}\left(-a b+c^{2}+2 c\right)^{2}\right]\right\} \\
& \left.\begin{array}{rl}
n=0, \quad q & =3 H+\sqrt{12 H^{2}-a^{2} I}, \\
q^{\prime} & =3 H-\sqrt{12 H^{2}-a^{2} I}
\end{array}\right\}, \\
& z_{1}^{*}=\sqrt{-q}, \quad z_{2}^{*}=-z_{1}^{*}, \quad z_{3}^{*}=\sqrt{-q^{\prime}}, \quad z_{4}^{*}=-z_{3}^{*}, \\
& s_{1}=\frac{z_{1}^{*}}{a}, \quad s_{2}=-s_{1}, \quad s_{3}=\frac{z_{3}^{*}}{a}, \quad s_{4}=-s_{3}, \\
& P_{L}=\frac{i k s_{L}(1+c)}{a s_{L}^{2}-p^{2}-k^{2}}=\frac{-s_{L}^{2}+p^{2}+b k^{2}}{-i k s_{L}(1+c)} \text { for } L=1,3 \\
& \text { and } \\
& P_{2}=-P_{1}, \quad P_{4}=-P_{3}
\end{aligned}
$$

From Eqs. (52), (53) and (66), we get the following relations for an orthotropic elastic medium

$$
\begin{aligned}
& \bar{u}(x, k)=C_{1} P_{1} e^{s_{1} x}-C_{2} P_{1} e^{-s_{1} x}+C_{3} P_{3} e^{s_{3} x}-C_{4} P_{3} e^{-s_{3} x}, \\
& \bar{v}(x, k)=C_{1} e^{s_{1} x}+C_{2} e^{-s_{1} x}+C_{3} e^{s_{3} x}+C_{4} e^{-s_{3} x}
\end{aligned}
$$

where $C_{1}, C_{2}, C_{3}$ and $C_{4}$ are constants to be determined from prescribed boundary conditions. From Eqs. (57)-(60) and (66) we get the following transformed stresses for an orthotropic medium

$$
\begin{aligned}
\bar{\tau}_{11}=[ & \left(a P_{1} s_{1}-i k c\right) C_{1} e^{s_{1} x}+\left(a P_{1} s_{1}-i k c\right) C_{2} e^{-s_{1} x} \\
& \left.+\left(a P_{3} s_{3}-i k c\right) C_{3} e^{s_{3} x}+\left(a P_{3} s_{3}-i k c\right) C_{4} e^{-s_{3} x}\right], \\
\bar{\tau}_{12}=[( & \left.-i k P_{1}+s_{1}\right) C_{1} e^{s_{1} x}+\left(i k P_{1}-s_{1}\right) C_{2} e^{-s_{1} x} \\
& \left.+\left(-i k P_{3}+s_{3}\right) C_{3} e^{s_{3} x}+\left(i k P_{3}-s_{3}\right) C_{4} e^{-s_{3} x}\right] .(68
\end{aligned}
$$

\section{Application: An Impulsive Line-load in an Infi- nite Orthotropic Elastic Medium}

We assume that a normal line-load is acting vertically downwards in the interior of an infinite orthotropic elastic medium. We shall determine the resulting displacements and stresses at any point of the medium. We take the $x$ axis vertically downwards and the given normal impulsive line-load $P_{0}$, per unit length, acting on the $z$-axis (Fig. 1). The elastic medium which occupies the region $x>0$ shall be called Medium-I and the region for which $x<0$ shall be called Medium-II. Medium-I and Medium-II have the identical elastic properties.

The boundary conditions for the displacements are

$$
\begin{aligned}
& u\left(x=0^{+}, y, t\right)=u\left(x=0^{-}, y, t\right), \\
& v\left(x=0^{+}, y, t\right)=v\left(x=0^{-}, y, t\right),
\end{aligned}
$$

and for stresses are

$$
\begin{aligned}
& \tau_{12}\left(x=0^{+}, y, t\right)=\tau_{12}\left(x=0^{-}, y, t\right), \\
& \tau_{11}\left(x=0^{+}, y, t\right)-\tau_{11}\left(x=0^{-}, y, t\right)=-P_{0} \delta(y) \delta(t) .
\end{aligned}
$$

Here, $\delta(y)$ is Dirac delta function, having the following properties,

$$
\int_{-\infty}^{\infty} \delta(y) d y=1, \delta(y)=\frac{1}{2 \pi} \int_{-\infty}^{\infty} e^{-i k y} d k, \quad F[\delta(y)]=1 .
$$

We define the dimensionless force $P_{0}^{\prime}$ as

$$
P_{0}^{\prime}=\frac{P_{0}}{d_{66}}
$$

We first write Eqs. (69) to (72) in non-dimensional forms and then after suppressing the dashes, apply the Laplace and Fourier transforms. We find

$$
\begin{aligned}
& \bar{u}\left(0^{+}, k, p\right)=\bar{u}\left(0^{-}, k, p\right), \\
& \bar{v}\left(0^{+}, k, p\right)=\bar{v}\left(0^{-}, k, p\right), \\
& \bar{\tau}_{12}\left(0^{+}, k, p\right)=\bar{\tau}_{12}\left(0^{-}, k, p\right), \\
& \bar{\tau}_{11}\left(0^{+}, k, p\right)-\bar{\tau}_{11}\left(0^{-}, k, p\right)=-P_{0} .
\end{aligned}
$$

The coefficients $C_{1}$ and $C_{3}$ in Eqs. (67) and (68) must be zero for the transformed displacements and stresses in 


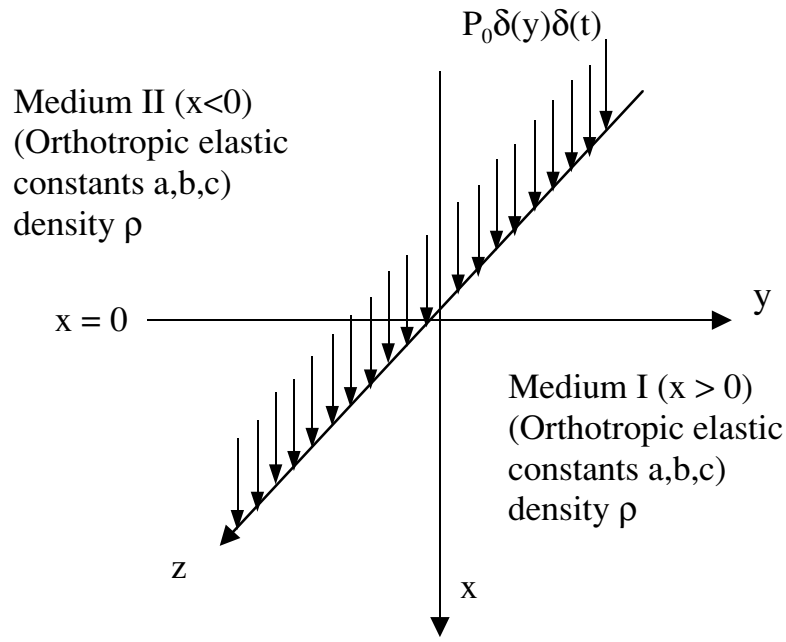

Fig. 1. An impulsive line-load on $z$-axis.

Medium-I. Therefore, transformed displacement and stress components in Medium-I, are

$$
\begin{aligned}
\bar{u} & =\left[-C_{2} P_{1} e^{-s_{1} x}-C_{4} P_{3} e^{-s_{3} x}\right], \\
\bar{v} & =\left[C_{2} e^{-s_{1} x}+C_{4} e^{-s_{3} x}\right], \\
\bar{\tau}_{11} & =\left[\left(a P_{1} s_{1}-i k c\right) C_{2} e^{-s_{1} x}+\left(a P_{3} s_{3}-i k c\right) C_{4} e^{-s_{3} x}\right], \\
\bar{\tau}_{12} & =\left[\left(i k P_{1}-s_{1}\right) C_{2} e^{-s_{1} x}+\left(i k P_{3}-s_{3}\right) C_{4} e^{-s_{3} x}\right] .
\end{aligned}
$$

Similarly, transformed displacement and stress components in Medium-II, are

$$
\begin{aligned}
\bar{u} & =\left[C_{1} P_{1} e^{s_{1} x}+C_{3} P_{3} e^{s_{3} x}\right], \bar{v}=\left[C_{1} e^{s_{1} x}+C_{3} e^{s_{3} x}\right], \\
\bar{\tau}_{11} & =\left[\left(a P_{1} s_{1}-i k c\right) C_{1} e^{s_{1} x}+\left(a P_{3} s_{3}-i k c\right) C_{3} e^{s_{3} x}\right], \\
\bar{\tau}_{12} & =\left[\left(-i k P_{1}+s_{1}\right) C_{1} e^{s_{1} x}+\left(-i k P_{3}+s_{3}\right) C_{3} e^{s_{3} x}\right] .
\end{aligned}
$$

Applying the boundary conditions in (73) and (74), we get the following values of the coefficients $C_{1}, C_{2}, C_{3}$ and $C_{4}$,

$$
C_{1}=-C_{2}=-C_{3}=C_{4}=\frac{P_{0}}{2 a\left(P_{1} s_{1}-P_{3} s_{3}\right)} .
$$

Thus, using Eq. (79), Equations (75)-(78) give the dimensionless displacement and stress components in the LaplaceFourier transformed domain in an infinite orthotropic elastic medium due to an internal impulsive line-load.

With the help of relations in Eq. 6(b) for a transversely isotropy, we obtain the solution of the corresponding problem for transversely isotropic elastic medium. Since, we have considered two-dimensional plane strain problem, parallel to $x y$-plane, the case of transversely isotropy coincides with that of perfectly isotropic elastic medium.

To get the displacement and stress components in the physical domain, we require to invert the Laplace and Fourier integrals. We invert them numerically.

\section{Inversion Techniques}

To get the function $f(x, y, t)$ in the physical domain from $\bar{f}(x, k, p)$, first we invert the Fourier transform by using

$$
\begin{aligned}
\hat{f}(x, y, p) & =\frac{1}{2 \pi} \int_{-\infty}^{\infty} \bar{f}(x, k, p) e^{-i k y} d k \\
& =\frac{1}{\pi} \int_{0}^{\infty}\left\{\bar{f}_{e} \cos k y-i \bar{f}_{0} \sin k y\right\} d k
\end{aligned}
$$

where $\bar{f}_{e}$ and $\bar{f}_{0}$ are even and odd parts of the function $\bar{f}(x, k, p)$, respectively. Thus expression (80) gives us the Laplace transform $\hat{f}(x, y, p)$ of the function $f(x, y, t)$. Now, the function $\hat{f}(x, y, p)$ in the expression (80) can be considered as the Laplace transform $\hat{g}(p)$ of some function $g(t)$. Following Honig and Hirdes (1984), the Laplace transformed function $\hat{g}(p)$ can be inverted numerically as outlined below.

The function $g(t)$ can be obtained from $\hat{g}(p)$ by using the relation

$$
g(t)=\frac{1}{2 \pi i} \int_{C-i \infty}^{C+i \infty} e^{p t} \hat{g}(p) d p
$$

where $C$ is an arbitrary real number greater than all the real parts of the singularities of $\hat{g}(p)$. Taking $p=C+i y$, we get

$$
g(t)=\frac{e^{C t}}{2 \pi} \int_{-\infty}^{\infty} e^{i t y} \hat{g}(C+i y) d y,
$$

Now, taking $e^{-C t} g(t)$ as $h(t)$ and expanding it as Fourier series in $[0,2 L]$, we obtain approximately by the formula

$$
g(t)=g_{\infty}(t)+E_{D},
$$

where

$$
\begin{aligned}
& g_{\infty}(t)=\frac{C_{0}}{2}+\sum_{k=1}^{\infty} C_{k}(t), 0 \leq t \leq 2 L, \\
& C_{k}=\frac{e^{C t}}{L} \operatorname{Re}\left[e^{\frac{i k \pi t}{L}} \hat{g}\left(C+\frac{i k \pi}{L}\right)\right],
\end{aligned}
$$

$E_{D}$ is called the discretization error and it can be made arbitrarily small by choosing $C$ large enough.

Since the infinite series in Eq. (84) can be summed up only to a finite number of terms, say $N$, so the approximate value of $g(t)$ becomes

$$
g_{N}(t)=\frac{C_{0}}{2}+\sum_{k=1}^{N} C_{k}(t), \quad 0 \leq t \leq 2 L,
$$

and

$$
E_{T}(t)=\sum_{k=N+1}^{\infty} C_{k}(t)
$$

is termed as truncation error. The truncation error must be added to the discretization error to produce the total approximate error in evaluating $g(t)$ using the above procedure.

For rapid convergence, the discretization error is reduced by using the Korrecktur method and then the $\in$-algorithm is used to reduce the truncation error.

The Korrecktur method formula, to evaluate the function $g(t)$ is

$$
g(t)=g_{\infty}(t)-e^{-2 C L} g_{\infty}(2 L+t)+E_{D}^{\prime},
$$

where

$$
\left|E_{D}^{\prime}\right| \ll\left|E_{D}\right| .
$$

Thus, the approximate value of $g(t)$ becomes

$$
g_{N_{k}}(t)=g_{N}(t)-e^{-2 C L} g_{N^{\prime}}(2 L+t),
$$




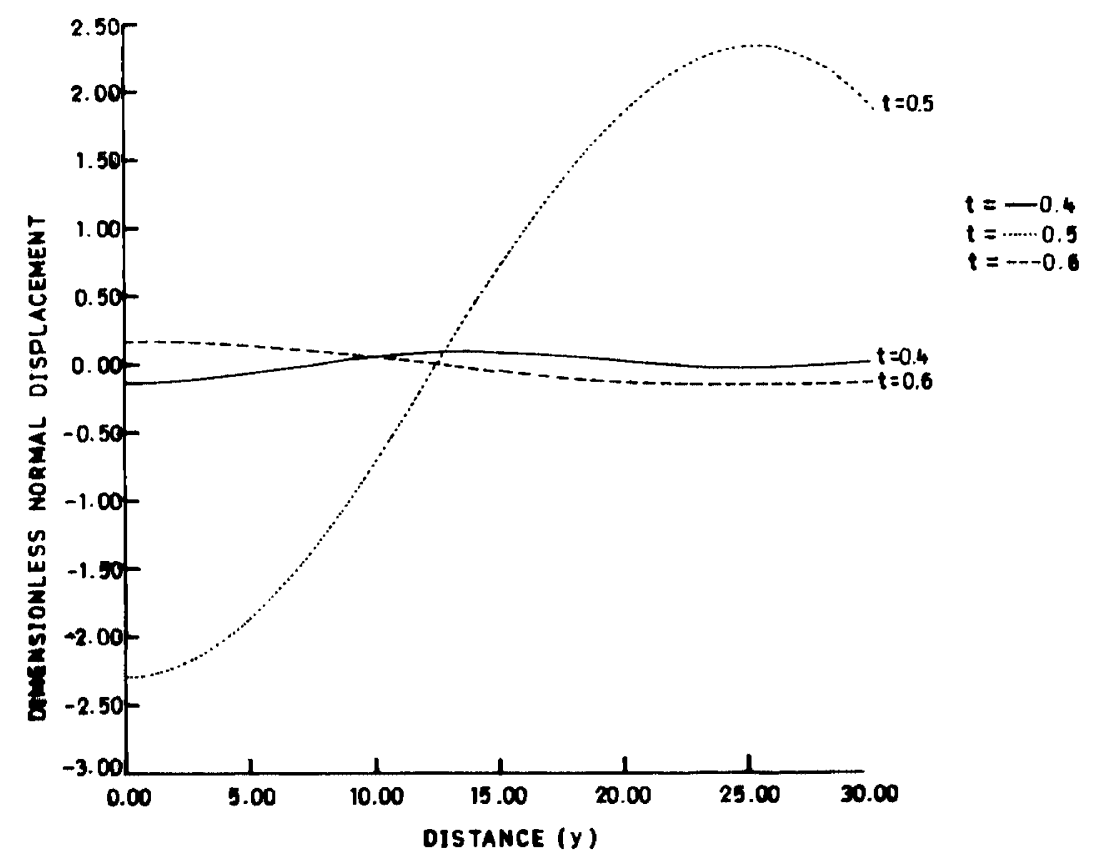

Fig. 2. Dimensionless normal displacement distribution on the plane $x=1.0$ for an orthotropic medium.

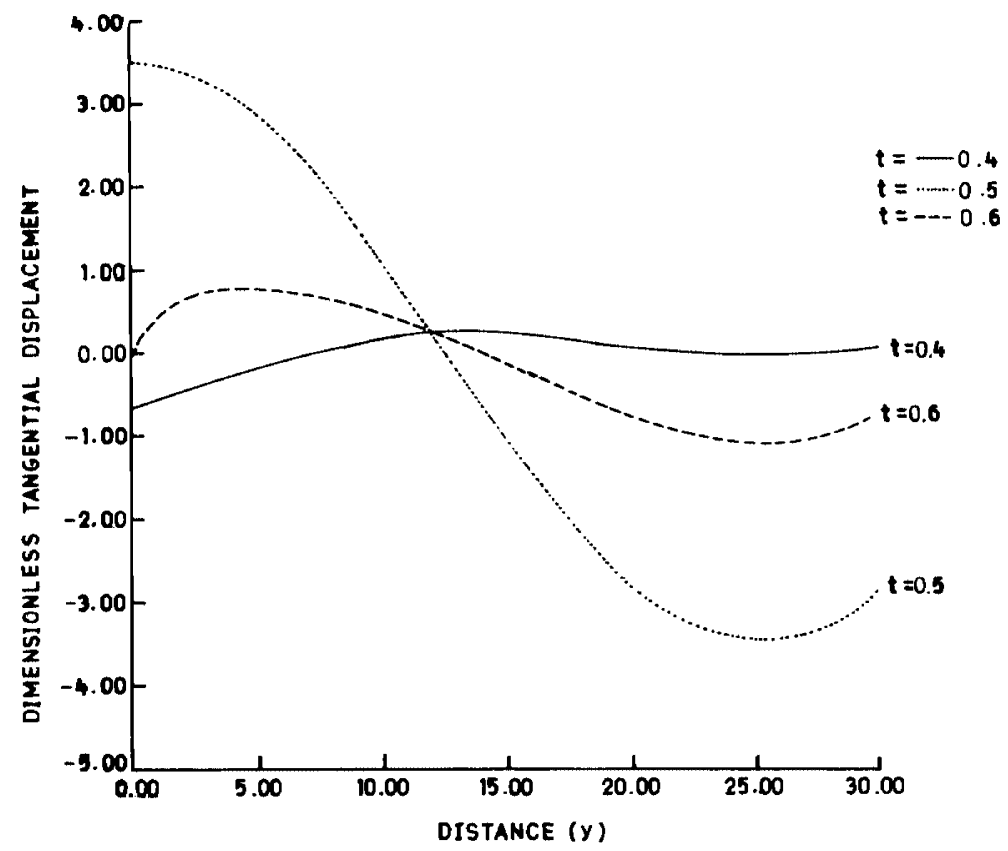

Fig. 3. Dimensionless tangential displacement distribution on the plane $x=1.0$ for an orthotropic medium.

where $N^{\prime}$ is an integer such that $N^{\prime}<N$.

We shall now describe the $\in$-algorithm which is used to accelerate the convergence of series (85). Let $N$ be an odd natural number and

$$
S_{m}=\sum_{k=1}^{m} C_{k}(t)
$$

be the sequence of partial sum of Eq. (85) and $\in$-sequence be defined as

$$
\begin{aligned}
& \epsilon_{0, m}=0, \quad \epsilon_{1, m}=S_{m}, \\
& \epsilon_{n+1, m}=\epsilon_{n-1, m+1}+\frac{1}{\epsilon_{n, m+1}-\epsilon_{n, m}}, \quad n, m=1,2,3, \cdots .
\end{aligned}
$$

The sequence $\epsilon_{1,1}, \epsilon_{3,1} \cdots \in_{N, 1}$ converges to $g(t)+E_{D}-$ $C_{0} / 2$ faster than the sequence $\left\{S_{m}\right\}$ of partial sums. The actual procedure to invert the Laplace transform consists of Eq. (88) together with the $\in$-algorithm. The values of $C$ and $L$ are chosen according to the criteria outlined by Honig and Hirdes (1984). The method for evaluating the integral in Eq. (80) is described by Press et al. (1986), which involves the use of Romberg's integration with adaptive step size. It, also uses the results from successive refinements of the extended trapezoidal rule followed by extrapolation of the results to the limit when the step size tends to zero. 


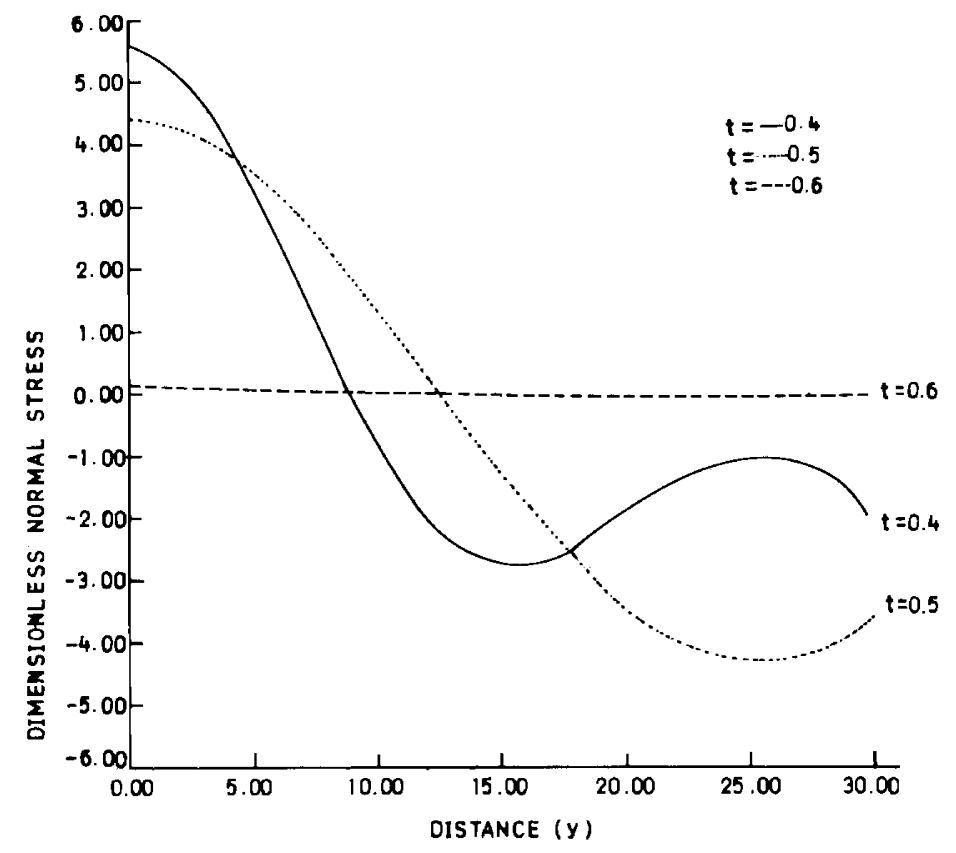

Fig. 4. Dimensionless normal stress distribution on the plane $x=1.0$ for an orthotropic medium.

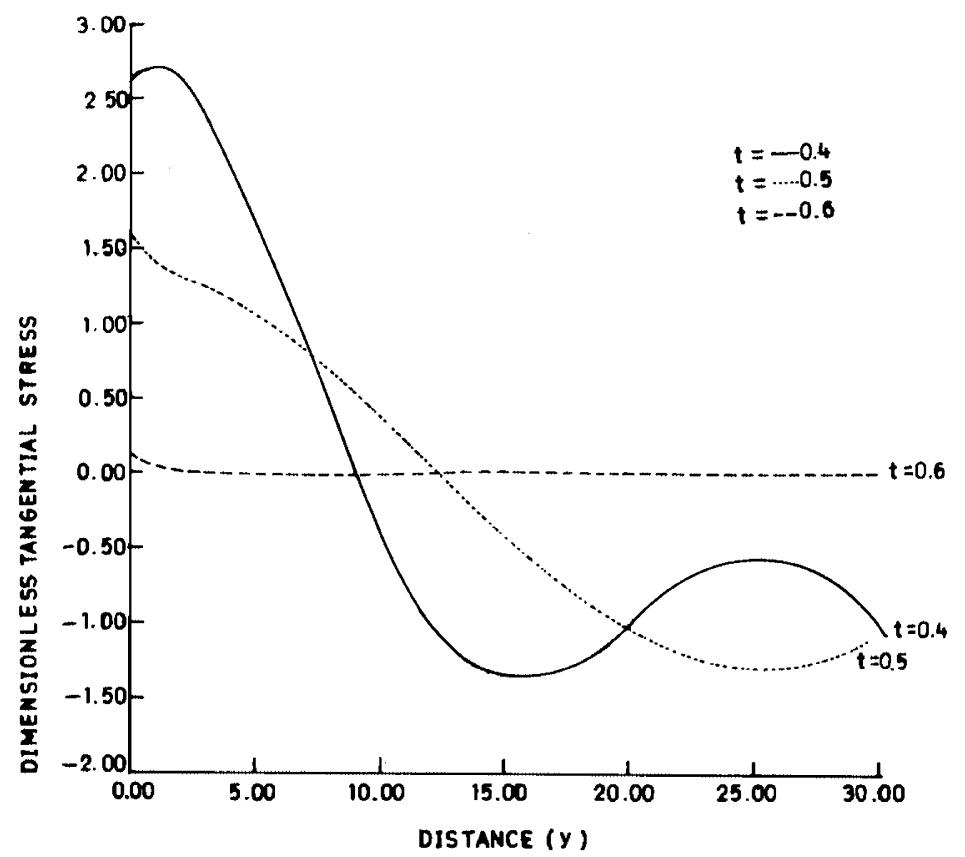

Fig. 5. Dimensionless tangential stress distribution on the plane $x=1.0$ for an orthotropic medium.

\section{Numerical Results and Discussion}

Using the numerical inversion techniques described in Section 4, the dimensionless displacement and stress components have been computed numerically in the time-domain. For numerical computation purposes, we use the following values of elastic constants given by Love (1944) for Topaz material

$$
\begin{aligned}
& d_{11}=2870, \quad d_{22}=3560, \quad d_{33}=3000 \\
& d_{12}=1280, \quad d_{23}=900, \quad d_{13}=860 \\
& d_{44}=1100, \quad d_{55}=1350, \quad d_{66}=1330
\end{aligned}
$$

in terms of a unit stress of $10^{6}$ grammes $\mathrm{wt} / \mathrm{cm}^{2}$. We assume that the dimensionless force $P_{0}$ is of unit magnitude.

The response represented by dimensionless displacement and stress component is calculated on the plane $x=1.0$, against horizontal distance ' $y$ ' for the different values of time $t$, namely $t=0.4,0.5$ and 0.6 . The distribution curves for an orthotropic medium are shown in Figs. 2-5. As the response, due to a normal line load, is symmetrical about the $x z$-plane, we have drawn various figures for the region $y \geq 0$. Figures 2-3 represent the distribution of dimensionless displacement components while Figures 4-5 represent the distribution of dimensionless stress components due to an impulsive 


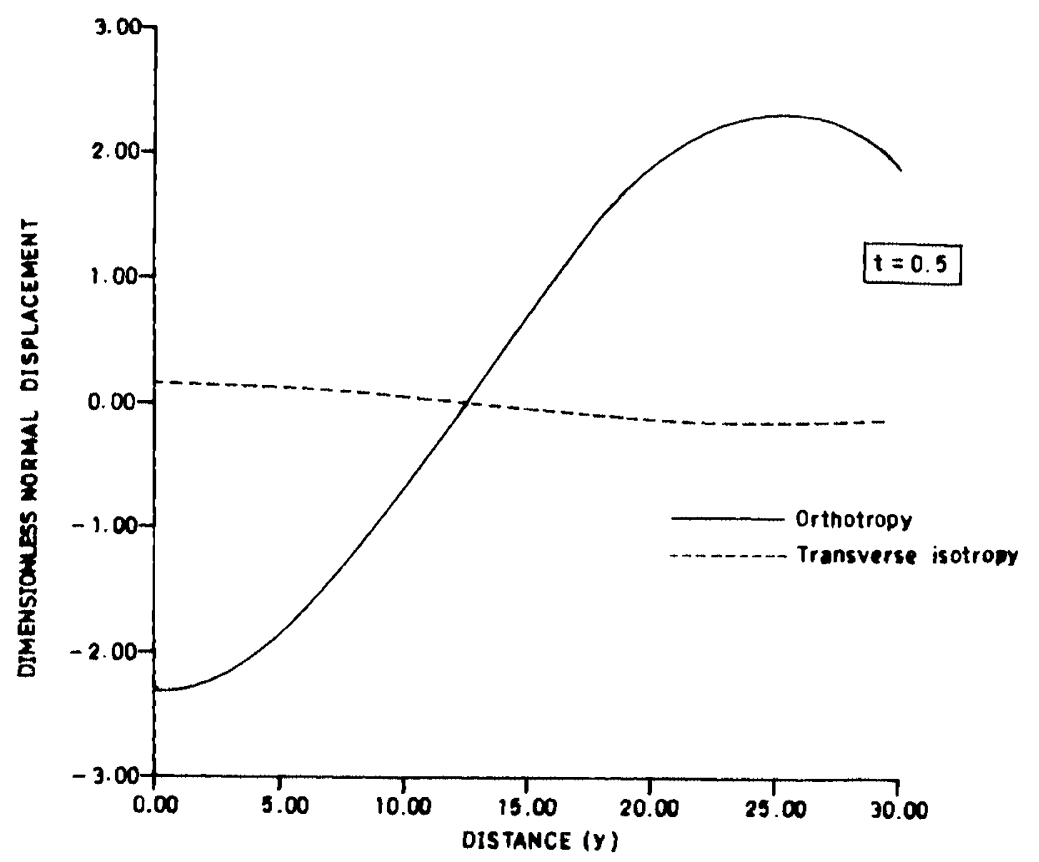

Fig. 6. Dimensionless normal displacement distribution on the plane $x=1.0$ for orthotropic and transversely isotropic media.

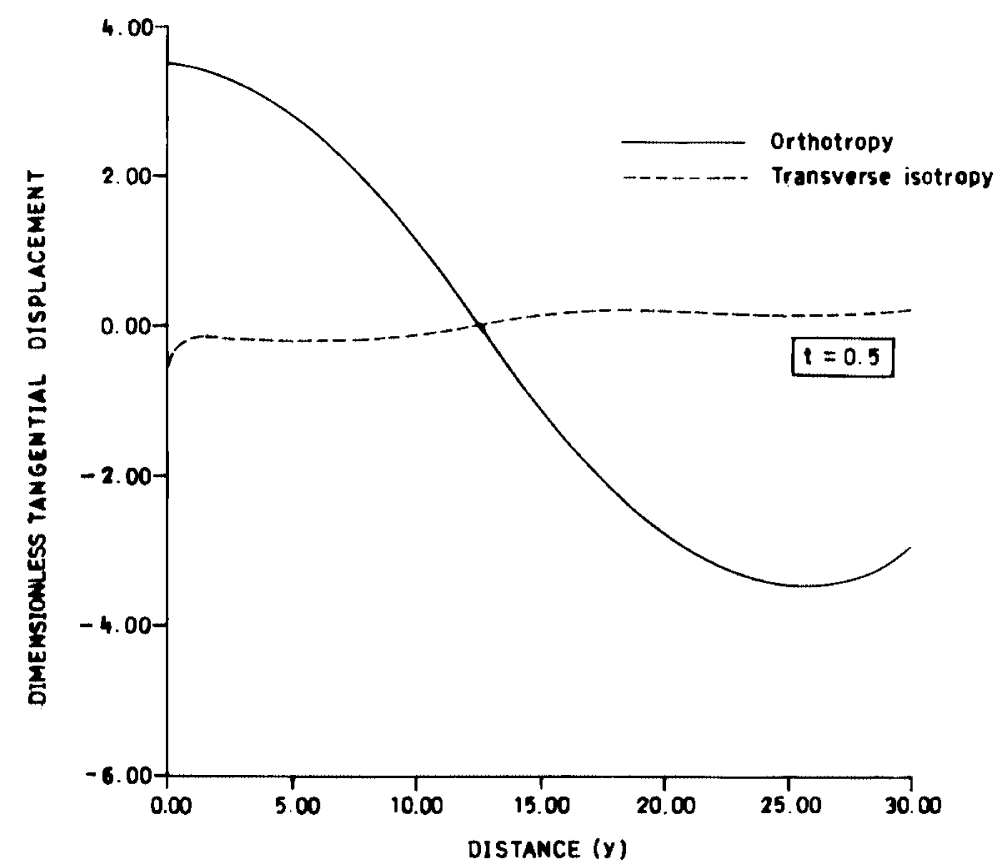

Fig. 7. Dimensionless tangential displacement distribution on the plane $x=1.0$ for orthotropic and transversely isotropic media.

source. The variation of the dimensionless normal displacement $\left(u / 10^{10}\right)$ is shown in Fig. 2, for three values of times, $t=0.4,0.5$ and 0.6 for an orthotropic medium. The variation of the dimensionless tangential displacement $\left(v / 10^{5}\right)$, corresponding to different values of $t$, is shown in Fig. 3. In Fig. 2, the dimensionless normal displacement corresponding to $t=0.4$ lies almost between the corresponding curves for $t=0.5$ and 0.6 whereas, in Fig. 3, the dimensionless tangential displacement corresponding to $t=0.6$ lies between the corresponding curves for $t=0.4$ and 0.5 . Three curves corresponding to different values of ' $t$ ' are plotted in Fig. 4 to show the variation of dimensionless normal stress $\left(\tau_{11} / 10^{11}\right)$. The variation of the dimensionless shear stress $\left(\tau_{12} / 10^{8}\right)$ is exhibited in Fig. 5 .

It is observed that with the increase in distance $y$ for each value of time $t$, the displacement and stress distribution curves for an orthotropic medium follows an oscillatory pattern and if we move further away (increase the value of $y$ more) they are approaching towards zero values. This observation justifies the radiation conditions used in the solution of the problem and this justification gives the numerical validation of the theoretical work done in the present paper.

To make the paper more interesting and to draw reader's attention, the effect of anisotropy is examined. Various dis- 


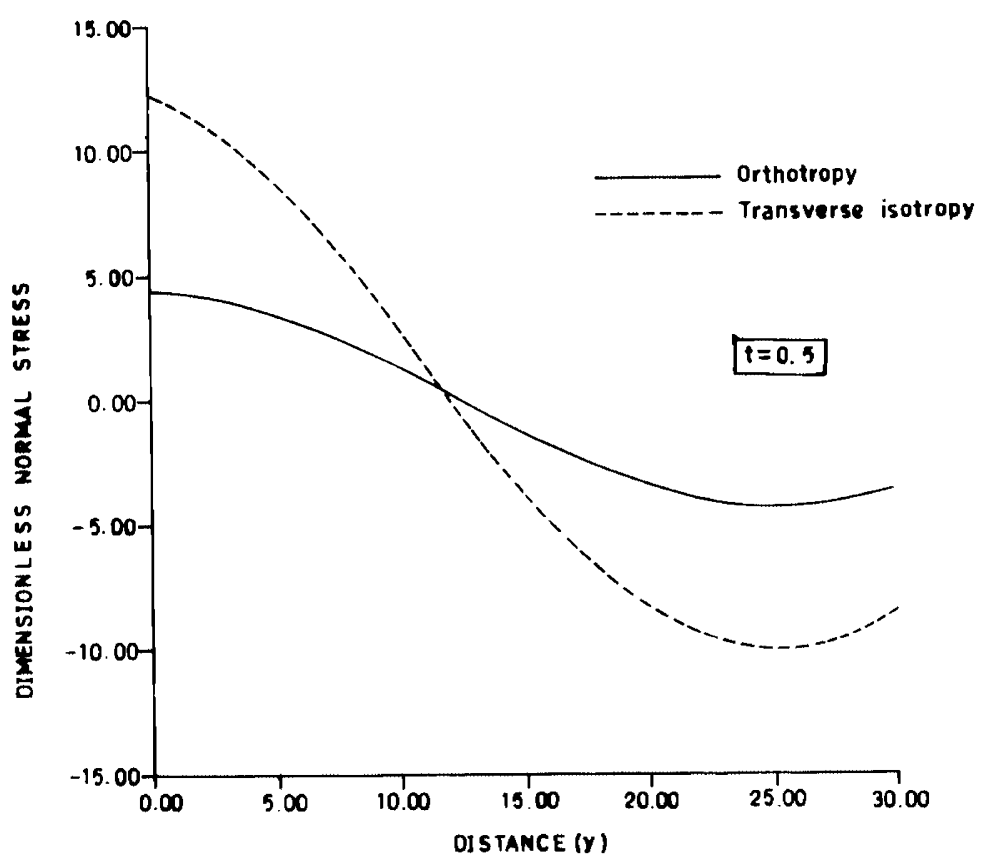

Fig. 8. Dimensionless normal stress distribution on the plane $x=1.0$ for orthotropic and transversely isotropic media.

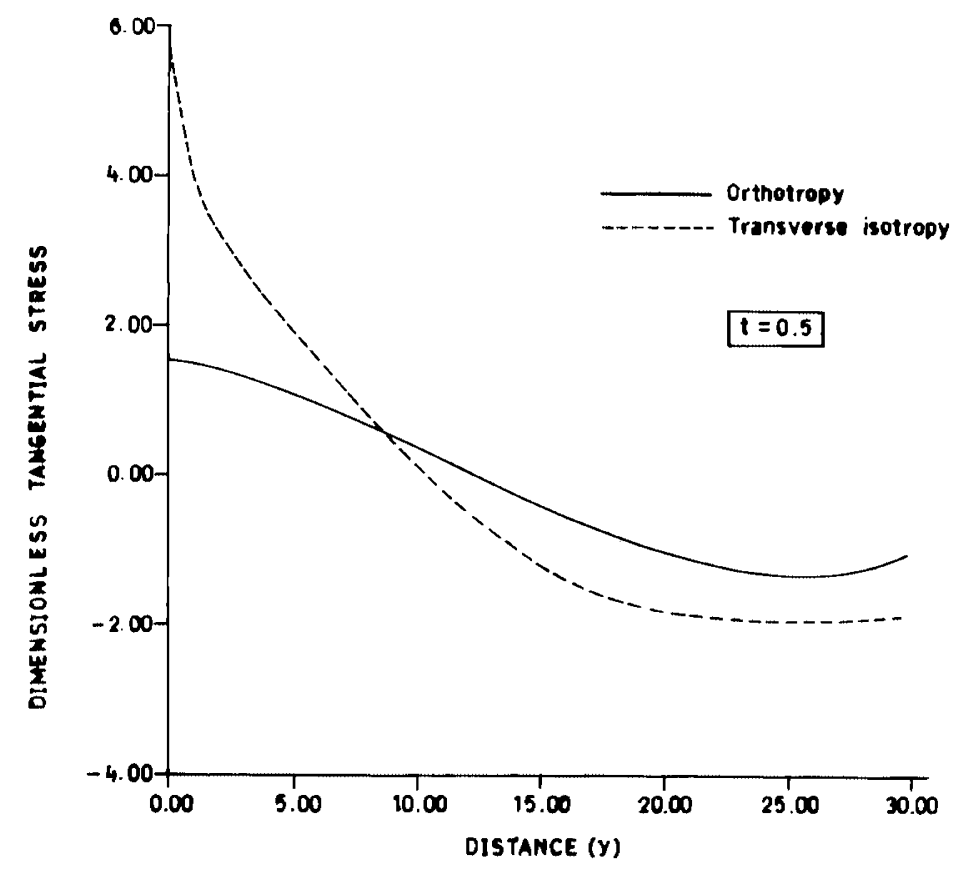

Fig. 9. Dimensionless tangential stress distribution on the plane $x=1.0$ for orthotropic and transversely isotropic media.

tribution curves are plotted for transversely isotropic and orthotropic elastic medium in Figs. 6-9. In these figures, distribution curves are drawn for orthotropic and transversely isotropic media for one fixed value of time $t=0.5$. While considering the above mentioned orthotropic medium as a transversely isotropic medium the following relations

$d_{22}=d_{11}, \quad d_{23}=d_{13}, \quad d_{55}=d_{44}, \quad d_{66}=\frac{1}{2}\left(d_{11}-d_{12}\right)$ are taken into consideration.

In Figs. 6-7 the variations of normal dimensionless displacements $\left(u / 10^{10}\right)$ and tangential dimensionless displace- ments $\left(v / 10^{5}\right)$ against the horizontal distance $(y)$ are shown. The variations of the normal dimensionless stress $\left(\tau_{11} / 10^{11}\right)$ and tangential dimensionless stress $\left(\tau_{12} / 10^{8}\right)$ are exhibited in Figs. 8-9.

It is evident from Figs. 6-9, that the orthotropy has significant effect on displacement and stress curves. Figures 6-7 show the effect on displacement distribution curves for transversely isotropic as well as orthotropic media and it is revealed that the magnitude of dimensionless normal and tangential displacement components are large for orthotropic medium in comparison to transversely isotropic medium. Further anisotropy is affecting the trend of distri- 
bution curves. Figures 8-9 for dimensionless stress distribution curves show that the effect of an anisotropy is mainly on magnitude but not on trend of the curves. Thus, it is concluded that the two types of anisotropy-transversely isotropy and orthotropy, have different behaviour due to the application of a normal line-load, and the difference is mainly quantitative in nature.

Acknowledgments. The authors are extremely grateful to the Reviewers (Prof. Bert Vermeersen and others) for giving their concrete and valuable suggestions which led the paper in the present form.

\section{References}

Achenbach, J. D., Wave Propagation in Elastic Solids, North-HollandElsevier, Amsterdam, 1973.

Atanackovic, T. M. and A. Guran, Theory of Elasticity for Scientist and Engineers, Birkhauser Boston, 2000.

Bonafede, M. and E. Rivalta, The tensile dislocation problem in a layered elastic medium, Geophys. J. Int., 136, 341-356, 1999a.

Bonafede, M. and E. Rivalta, On tensile cracks close to and across the interface between two welded elastic half-spaces, Geophys. J. Int., 138, 410-434, 1999b.

Buchwald, V. T., Elastic waves in anisotropic media, Proc. R. Soc. Lond., A253, 563-580, 1959.

Burridge, R., The singularity on the plane lids of the wave surface of elastic media with cubic symmetry, Q. J. Mech. Appl. Math., 20, 40-56, 1967.

Debnath, L. and Loknath, Integral Transforms and Their Application, CRC Press Inc., New York, 1995.

Duff, G. F. D., The Cauchy problem for elastic waves in an anisotropic medium, Phil. Trans. R. Soc. Lond., A252, 249-273, 1960.

Garg, N. R., D. K. Madan, and R. K. Sharma, Two-dimensional deformation of an orthotropic elastic medium due to seismic sources, Phys. Earth Planet. Inter., 94, 43-62, 1996.

Garg, N. R., R. Kumar, A. Goel, and A. Miglani, Plane strain deformation of an orthotropic elastic medium using an eigenvalue approach, Earth Planets Space, 55(1), 2003.

Hoing, G. and U. Hirdes, A method for the numerical inversion of the Laplace transform, J. Comp. Appl. Math., 10, 113-132, 1984.

Karabolis, D. L. and D. E. Beskos, Dynamic response of 3-D rigid surface foundations by time domain boundary element method, Earthquake Eng. Structure Dyn., 12, 73-93, 1984.

Lamb, H., On the propagation of tremors over the surface of an elastic solid, Phil. Trans. Roy. Soc. Am., 203, 1-42, 1904.

Lighthill, M. J., Studies on magneto-hydrodynamic waves and other anisotropic wave motions, Phil. Trans. R. Soc. Lond., A252, 397-430, 1960.

Love, A. E. H., A Treatise on the Mathematical Theory of Elasticity, Dover
Publications, New York, 1944.

Maruyama, T., On the force equivalents of dynamical elastic dislocations with reference to the earthquake mechanism, Bull. Earth. Res. Inst. (Tokyo), 41, 467-486, 1963.

Maruyama, T., On two-dimensional elastic dislocations in an infinite and semi-infinite medium, Bull. Earthq. Res. Inst., 44, 811-871, 1966.

Mase, G. T. and G. E. Mase, Continuum Mechanics for Engineers, CRC Press LLC., New York, 1999.

Mura, T., Micromechanics of Defects in Solids, 2nd edition, Kluwer Academic Publishers, London, 1987.

Niwa, Y., T. Fukui, S. Kato, and K. Fujiki, An application of the integral equation method to two-dimensional elastodynamics, in Theoretical and Applied Mechanics, vol. 28, University of Tokyo Press, Tokyo, pp. 281290, 1980.

Okada, Y., Surface deformation due to inclined shear and tensile faults in a homogenous isotropic half space, Bull. Seismol. Soc. Am., 75, 1135$1154,1985$.

Okada, Y., Internal deformation due to shear and tensile faults in a halfspace, Bull. Seismol. Soc. Am., 82, 1018-1040, 1992.

Pan, E., Static response of a transversely istropic and layered half space to general dislocation sources, Phys. Earth Planet. Inter., 58, 103-117, 1989.

Payton, R. G., Elastic Wave Propogation in Transversely Isotropic Media, Matrtinus Nijhoff Publishers, The Hague, 1983.

Piersanti, A., G. Spada, R. Sabadini, and M. Bonafede, Global post-seismic deformation, Geophys. J. Int., 120, 544-566, 1995.

Press, W. H., S. A. Teukolsky, W. T. Velleling, and B. P. Flannery, Numerical Recipes in Fortran, Cambridge University Press, Cambridge, 1986.

Ross, S. L., Differential Equations, 3rd edition, John Wiley and Sons Inc., 1984.

Sneddon, I. N., Fourier Transforms, McGraw-Hill, New York, 1951.

Stokes, G. G., On the dynamical theory of diffraction, Trans. Camb. Phil. Soc., 9, 1-62, 1849.

Tverdokhlebov, A. and J. Rose, On Green's function for elastic waves in anisotropic media, J. Acoust. Soc. Am., 83, 118-121, 1988.

Wang, C. Y. and J. D. Achenbach, A new look at 2-D time-domain elastodynamic Green's functions for general anisotropic solids, Wave Motion, 16, 389-405, 1992.

Wang, C. Y. and J. D. Achenbach, A new method to obtain 3-D Green's functions for anisotropic solids, Wave Motion, 18, 273-289, 1993.

Wang, C. Y. and J. D. Achenbach, Elastodynamic fundamental solutions for anisotropic solids, Geophys. J. Int., 118, 384-392, 1994.

Yeatts, F. R., Elastic radiation from a point source in an anisotropic medium, Phys. Rev. B, 29, 1674-1684, 1984.

N. R. Garg (e-mail: nrgmath@rediffmail.com), A. Goel, A. Miglani, and R. Kumar 